\title{
Data-Fitted Second-Order Macroscopic Production Models
}

Louis Forestier-Coste, Simone Göttlich and Michael Herty

Preprint No. 408

October 2014

Key words: production systems, system of conservation laws, data fitting

AMS Subject Classifications: 90B30, 35L65, 91B74

Institut für Geometrie und Praktische Mathematik

RWTH Aachen

Templergraben 55, D-52056 Aachen, (Germany)

\footnotetext{
(L. ForestierCoste) RWTH Aachen University

E-mail address: forestier@igpm.rwth-aachen.de

(S. Göttlich) University of Mannheim

E-mail address, Corresponding author: goettlich@uni-mannheim.de

(M. Herty) RWTH Aachen University

E-mail address: herty@igpm.rwth-aachen.de
} 


\title{
DATA-FITTED SECOND-ORDER MACROSCOPIC PRODUCTION MODELS
}

\author{
L. FORESTIER-COSTE, S. GÖTTLICH, AND M. HERTY
}

\begin{abstract}
Starting from discrete event simulations based on sampled data we simulate the interplay between product density and flux. Data-fitting helps to determine the right parameters for clearing functions to close first and second order conservation laws. For the first order case well-known relations from $\mathrm{M} / \mathrm{M} / 1$-queuing theory can be reproduced and numerically extended to transient behavior. To include more information from the data into the model, a second equation is introduced leading to a second order production model which is close to the Aw-Rascle-Zhang model known from traffic flow. Numerical comparisons show similarities and, in particular, differences of the modeling approaches.
\end{abstract}

AMS subject classifications: 90B30, 35L65, 91B74

Keywords: production systems, system of conservation laws, data fitting

\section{INTRODUCTION}

Recently, macroscopic or fluid-like models have been introduced to model high-volume product flow $[4,8,15,16,17,18,20,26]$. In some cases the fluid-like models have been derived as mean field limit $[4,7]$ to microscopic product dynamics. Those dynamics are inspired by discrete event simulations (DES), see [14]. The DES is a stochastic simulation for individual particles whereas the fluid-like continuous models describe the production flow in an aggregate way leading to coarse-grained and computationally efficient models. The proposed continuous models are conservation laws

$$
\partial_{t} \rho+\partial_{x} F(\rho)=0
$$

for the product density $\rho(x, t)$ at production stage $x \in[0,1]$ and time $t \geq 0$. Here, the flux function $F$ is called function. Starting with Graves [22] and Karmarkar [27] monotone, concave clearing functions have been proposed and are now used as modeling technique in production engineering, see for example [10,11,31]. Other approaches to derive clearing functions through mean field limit [4, 7] or by comparison with observed behavior [6] have been also studied. Under simplified assumptions the fundamental relation between $F$ and $\rho$ is obtained rigorously as steady-state by queuing theory. In the case of a single unlimited buffer, Poisson processes for the arrival of products and a Poisson process for the production time $\mathrm{M} / \mathrm{M} / 1$ one obtains $F=\frac{\mu W}{1+W}$, where $W=\int_{0}^{1} \rho(x, t) d x$ is the (total) work-in-progress (wip), see for example [25]. We may use $\rho$ and $W$ equivalently whenever $\rho$ is constant in $x$. Here, $\mu$ is the production rate and $F$ the arrival rate. In [31], a clearing function for an $M / G / 1$ is proposed including parameters that may adjusted to given data. The resulting clearing function is again a steady-state consideration and

Date: October 1, 2014. 
in general for models based on product flow no transient clearing function model has been derived [32]. It has been observed [30] that in different production periods different clearing functions may be suitable. We also refer to $[9,16]$ for an overview.

In this paper we propose a different approach using data to establish the clearing function, i.e., the fundamental relation between $F$ and $\rho$. This is inspired by recent progress in the field of traffic flow [19] where by comparing macroscopic traffic flow models to traffic data, new relations could be obtained. Existing projects in the field of traffic flow as the Mobile Millennium Project [1] and the Mobile Century project [3] focus on the assimilation of data and the reconstruction of traffic states. In contrast, here we derive new macroscopic models based on sampled data in order to predict production behavior. Additionally, we extend the usually used scalar clearing function $F: \mathbb{R} \rightarrow \mathbb{R}$ to a system of hyperbolic equations. In view of the terminology in traffic flow the arising family of clearing functions $F: \mathbb{R}^{2} \rightarrow \mathbb{R}^{2}$ is viewed as a second-order model for production. Those comprise of systems of hyperbolic equations in two variables, the product density $\rho=\rho(x, t)$ and a product property called $Y=Y(x, t)$. From a mathematical point of view the arising system of hyperbolic equations inherits features of the second-order Aw-Rascle-Zhang traffic flow model. Those have been introduced in $[13,33,36]$ and in a discretized setting earlier in [28] and extended by many authors as for example [19, 24]. As discussed previously non-stationary queuing theory predicts that there is no fixed functional relationship between product density and flux [32]. However, transient clearing functions have been proposed starting with the work of [2] and [34] to incorporate dynamic effects. Here, we propose a different way to include transient behavior. Since we are interested in the qualitative behavior of aggregate product flow on possibly large-time scales, we try to capture transient behavior by introducing a family of clearing functions and by relying on the dynamics induced by the system of hyperbolic partial differential equations.

A different approach towards second-order models has been discussed in [5]. Therein, the fluid model hierarchy proposed in [7] has been used to simulate transient influx. Compared with the presented approach a discussion of theoretical properties as well as a comparison to a DES has not been conducted. We discuss further differences after deriving the model in Remark 1 below.

We consider a setup as in $[4,16,20]$ where a high-volume multi-stage production line is considered. The quantity of interest is the product or lot density $\rho(x, t)$ describing the work-in-progress at time $t$ and production stage $x$. Here, $x \in[0,1]$ does not represent a physical position but rather the degree of completion or stage of production. The factory has a prescribed inflow $\lambda(t)$ over time $t$ at $x=0$ and an outflow at $x=1$ of finished products. Since mass conservation holds true the system fulfills the conservation law

$$
\partial_{t} \rho(x, t)+\partial_{x} F(\rho(x, t))=0, \quad x \in[0,1], \quad t \geq 0 .
$$

The equation (1) is accompanied by initial data $\rho(x, 0)=\rho_{0}(x)$ describing the initial state of the factory as well as a boundary condition $F=\lambda(t)$ at $x=0$. The paper is concerned with the detailed data-fitted modeling of the flux function $F$. The discussion includes well-posedness of the boundary condition at $x=0$. The model (1) is based on the assumption that the amount of products and the number of production stages justifies a continuous model. A prototype of a production process consists of a machine with associate buffer. In case of an $\mathrm{M} / \mathrm{M} / 1$-process the machine is fed by an arrival 
process with exponentially distributed inter-arrival times (with mean $\lambda$ ). There is no limit on the storage capacity of the buffer. The average processing rate of the machine is $\mu$. In case of constant rates elementary queuing theory gives the mean cycle-time $\tau=\frac{1}{\mu-\lambda}$ and Little's law $\rho=\tau \lambda$, where the constant $\rho$ is the average number of parts. In case of constant product density $\rho$, the total work-in-progress $W$ fulfills $W=\rho$. This leads to the relation $\lambda=\frac{\mu \rho}{1+\rho}$ and the previously mentioned clearing function $F=F(\rho)=\frac{\mu \rho}{1+\rho}$.

As depicted in Figure 1, a DES taken for example as explained in the Appendix A leads to an average density and flux relations fulfilling the $\mathrm{M} / \mathrm{M} / 1$-process. However, each single evaluation of the DES yields a data point in the wip-flux diagram depicted in Figure 1. For large number of parts and many realizations this resembles clearly the known relation. We try to capture now time-dependent queuing by fitting a family of clearing functions to the given diagram in Figure 1. This procedure may also be applied if for example the data depicted in Figure 1 is obtained not by a DES simulation but by measured data.

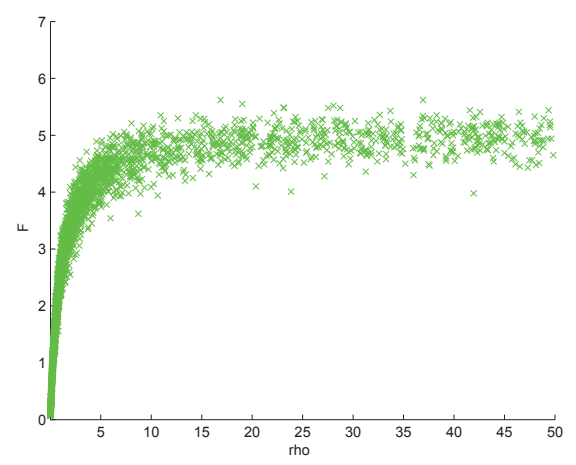

Figure 1. Discrete event simulation as described in Appendix A in log-scale to emphasize the behavior at low product densities for fixed production rate $\mu=5$. The inter arrival rate $\lambda=F$ is fixed for every DES simulation but varying over a range from $0 \leq \lambda \leq \mu$. Each green dot represents a single DES simulation. At each simulation the total work-in-progress $W$ is constant and equal to the constant production density $\rho$. Time horizon for the DES is $T=10^{3}$ and approximately $10^{6}$ simulations have been performed.

\section{DATA-FITTED CLEARING FAMILY OF CLEARING FUNCTIONS}

The starting point for our investigations is a DES leading to the relation of the flux $F$ and the product density $\rho$ discussed above and depicted in Figure 1 and Figure 2, respectively. In the $\mathrm{M} / \mathrm{M} / 1$ case this leads to the equation

$$
F(\rho)=\mu \frac{\rho}{1+\rho}
$$

with production rate $\mu$. Obviously, the graph fits the average of sampled data points very well but misses the spread of the data. This is of course a generic problem of scalar models comprising of a single function used to fit widely scattered data. 

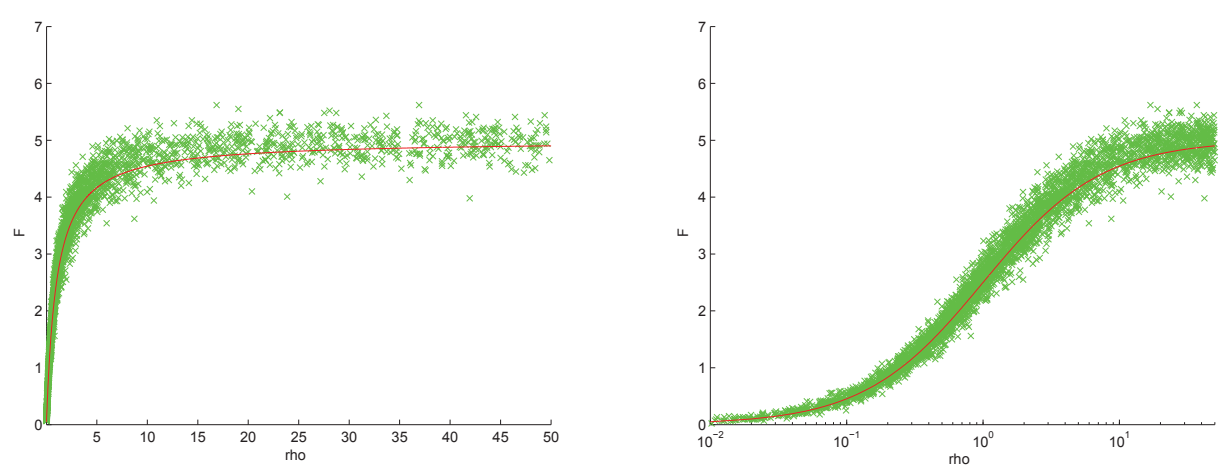

Figure 2. The setting is the same as in Figure 1. The solid (red) line shows the function (2) for $\mu=5$.

A possible remedy is to fit not a single function, but a family. An example of a family of clearing functions is depicted in Figure 3 covering the full range of existing data. The family is similar to equation (2) but using a variable production rate. To distinguish the two approaches we introduce a new variable $Y>0$ and consider the clearing function depending on $\rho$ and $Y$ as

$$
F(\rho, Y)=\frac{Y \rho}{1+\rho}
$$
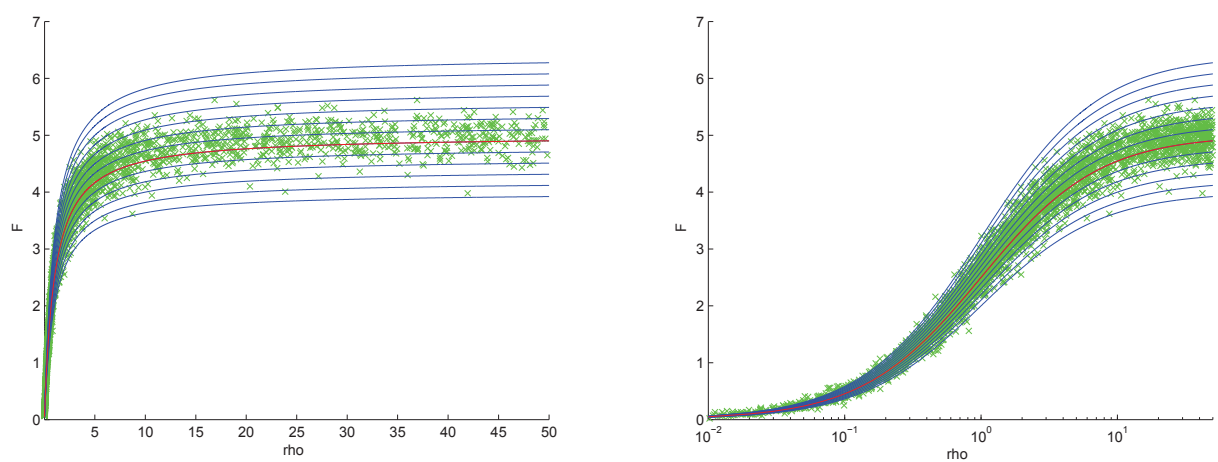

Figure 3. The setting is the same as in Figure 2. The blue lines represent the graph of the function given in equation (3) for different fixed values of $Y$.

Clearly, equation (1) together with equation (3) do not form a closed system. In order to derive an equation for the dynamics of the new quantity $Y$ we assume as in the AwRascle-Zhang model $[13,36]$ that the information $Y$ is transported with the velocity of the products. This means that $Y$ is essentially a product property propagating in direction of the stage of completion $x$. The (average) velocity $u$ of the product is given 
by

and the equation for $(\rho, Y)$ therefore reads

$$
u=\frac{F(\rho, Y)}{\rho}=\frac{Y}{1+\rho}, \quad \rho>0
$$

$$
\begin{aligned}
& \partial_{t} \rho+\partial_{x}(\rho u)=0, \\
& \partial_{t} Y+u \partial_{x} Y=0, \quad \text { with } \quad Y=(1+\rho) u .
\end{aligned}
$$

The system (4) may be written in conservative form and we show below that it is a well-posed system of hyperbolic conservation laws. Due to the close relation to the AwRascle-Zhang model we refer to equation (4) as the second-order model. The clearing function $F=\rho u=\frac{Y \rho}{1+\rho}$ is given by the family of functions (3) obtained by data-fitting to a DES and using the additional assumption that the newly introduced property $Y$ propagates in direction of the stage $x$ by the average product velocity $u$.

We also derive below that the first family of Riemann invariants of (4) are graphs $\rho \rightarrow F(\rho, Y)$ for a fixed value of $Y$ as depicted in Figure 3.

This implies that for initial data with constant $Y$ the predictions of production density $\rho$ given by equation (4) coincide with those obtained by equation (1) and equation (2). Hence, equation (4) extends the previously introduced models. Furthermore, we note that in the Aw-Rascle-Zhang system the relation between $Y$ and $\rho$ has been modeled as $Y=u+p(\rho)$ for some $p \geq 0$ with $p(\rho)=\rho^{\gamma}, \gamma>0$. Due to the different relation between $u, \rho$ and $Y$ the system (4) enjoys some fundamentally different properties compared with the Aw-Rascle-Zhang type model.

\section{Mathematical Properties of the SECOND-ORDER MOdeL}

In order to analyze the properties of the model we reformulate equation (4) in conservative form. We only briefly discuss those properties due to the similarity with the discussion in [13]. The conservative variables are $\rho$ and $z=u \rho(1+\rho)=\rho Y$, i.e., the product density and the weighted product property. Then, the second-order model reads

$$
\begin{aligned}
& \partial_{t} \rho+\partial_{x}(\rho u)=0, \\
& \partial_{t} z+\partial_{x}(z u)=0, \quad \text { with } \quad u=u(\rho, z)=\frac{z}{\rho(1+\rho)} .
\end{aligned}
$$

The flux function for the system (5) is given by

$$
f(\rho, z)=\left(\begin{array}{c}
\rho u \\
z u
\end{array}\right), \quad u(\rho, z)=\frac{z}{\rho(1+\rho)} .
$$

The Jacobian of the flux function $f(\rho, z)$ is

$$
D f(\rho, z)=\left(\begin{array}{cc}
-\frac{z}{(\rho+1)^{2}} & \frac{1}{\rho+1} \\
-\frac{z^{2}(2 \rho+1)}{\rho^{2}(\rho+1)^{2}} & \frac{2 z}{\rho(\rho+1)}
\end{array}\right)=\left(\begin{array}{cc}
-\frac{u \rho}{\rho+1} & \frac{1}{\rho+1} \\
-u^{2}(2 \rho+1) & 2 u
\end{array}\right)
$$

and its eigenvalues are

$$
\lambda_{1}(\rho, z)=\frac{z}{\rho^{3}+2 \rho^{2}+\rho}=\frac{u}{1+\rho}, \quad \lambda_{2}(\rho, z)=\frac{z}{\rho(1+\rho)}=u .
$$

As modeled the speed of information propagation is bounded by the velocity of the products $u$ which seems a reasonable assumption for production processes, i.e., $\lambda_{1}(\rho, z) \leq$ 
$\lambda_{2}(\rho, z)$. Except for vacuum $\rho=0$, where formally also $f$ is not defined, the eigenvalues are strictly separated and the system is strictly hyperbolic.

We compute the associated eigenvectors and consider further features of the system (5). The eigenvectors are

$$
r_{1}(\rho, z)=\left(\begin{array}{c}
\frac{1}{u(1+\rho)} \\
1
\end{array}\right), \quad \text { and } \quad r_{2}(\rho, z)=\left(\begin{array}{c}
\frac{1}{u(2 \rho+1)} \\
1
\end{array}\right) .
$$

Hence, the first characteristic field is genuine nonlinear $\nabla \lambda_{1} \cdot r_{1} \neq 0$ and the second characteristic field is nonlinearly degenerated $\nabla \lambda_{2} \cdot r_{2}=0$ for all $(\rho, z)$. The system is a Temple system. The second field leads to a contact discontinuity traveling at speed $u$. The first field has coinciding shock and rarefaction waves. The Rankine-Hugeniot condition is given by

The Riemann invariants are

$$
s \frac{\rho-\rho^{*}}{z-z^{*}}=\frac{\rho u-\rho^{*} u^{*}}{z u-z^{*} u^{*}} .
$$

$$
w_{1}:=\frac{z}{\rho}=u(1+\rho) \text { and } w_{2}:=\frac{z}{\rho(1+\rho)} .
$$

The Riemann invariants allow to simply depict the wave curves in the $\rho-\rho u$ plane. The first family is given by $w_{1}=$ const and the second family by $w_{2}=$ const. Therefore, all points lying on the second family fulfill

$$
\text { const }=w_{2}=\frac{z}{\rho(1+\rho)}=u
$$

and hence in the plane $(\rho, \rho u)$ the second family comprises of a straight line with slope given by a constant $u^{*}$. Similarly, for the first family we obtain

$$
\rho \text { const }=\rho w_{1}=\text { const } \frac{\rho}{1+\rho} \text {. }
$$

And for a constant $u^{*}\left(1+\rho^{*}\right)$ we get

$$
\left\{(\rho, \rho u):(\rho u)=\left(u^{*}\left(1+\rho^{*}\right) \frac{\rho}{1+\rho}\right\} .\right.
$$

As expected this is precisely $F\left(\rho, Y^{*}\right)$ given by (3) for the constant $Y^{*}=u^{*}\left(1+\rho^{*}\right)$. Having the wave curves at hand, the solution to the Riemann problem is immediate. In general for non-vacuum data, it may consist of a superposition of a (slow) rarefaction or shock wave with $w_{2}=c s t$ and a (fast) contact discontinuity with $w_{1}=c s t$. For a left state $\left(\rho_{\ell}, z_{\ell}\right)$ and a given right state $\left(\rho_{r}, z_{r}\right)$ we obtain a shock wave if $\rho_{\ell}<\rho_{r}$ and $w_{2}\left(\rho_{\ell}, z_{\ell}\right)=w_{2}\left(\rho_{r}, z_{r}\right)$ of speed $s=\frac{\rho_{\ell} z_{\ell}-\rho_{r} z_{r}}{\rho_{\ell}-\rho_{r}}$. In the other case we obtain a rarefaction wave. The construction is similar to the Aw-Rascle-Zhang model and therefore not repeated here. The reader is referred to [13, Section 2]. Hence, apart from vacuum $\rho=0$ the system (5) is a strictly hyperbolic system of Temple type. The wave curves are depicted in Figure 4.

In the $\rho-\rho u$ plane the graph of the Riemann invariants for the first family $w_{1}=$ const $>0$ are concave and monotone increasing functions. Therefore, all waves of the first family have non-negative speed provided that $\rho>0$ and $u>0$, respectively. This is different compared with traffic flow models but in accordance with the expected behavior of a production line. No information may travel backwards in the production line which 

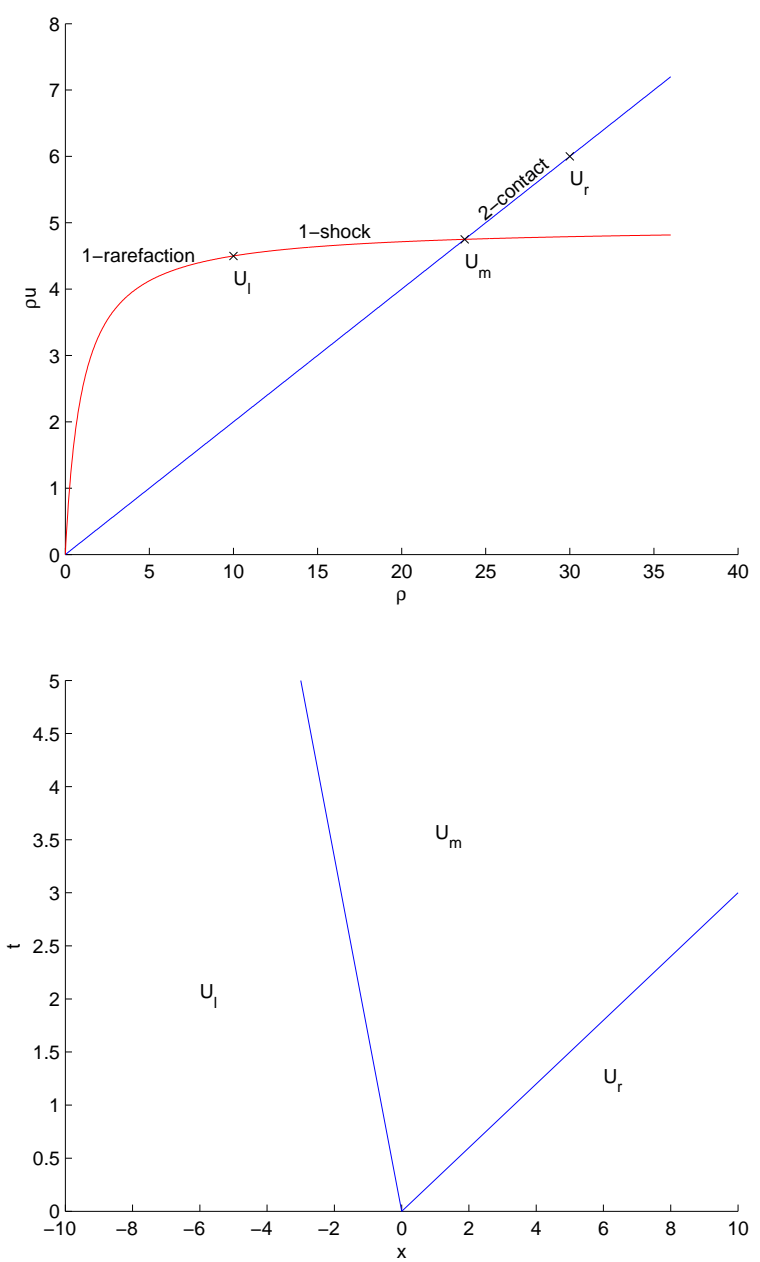

Figure 4. Above: Wave curves of the hyperbolic system (5) in the $\rho-$ $\rho u$ plane. The state $U_{l}=\left(\rho_{l}, \rho_{l} u_{l}\right)$ is the constant left initial datum of a Riemann problem and the state $U_{r}$ the right initial datum. The intermediate state is denoted by $U_{m}$. Below: The corresponding picture in the $x-t$ plane in case of a shock wave, i.e., $\rho_{l}<\rho_{r}$, is given.

resembles the fact that we have an infinite buffer resulting in the data of Figure 1. In case of finite buffers we expect a different behavior as discussed in [6].

Remark 1. The presented model (4) may also be compared with a second order model proposed in [5] restated here for convenience

$$
\partial_{t} \rho+\partial_{x}(\rho u)=0 \text { and } \partial_{t} u+u \partial_{x} u=0 .
$$

The model (6) coincides with pressure-less gas dynamics and the dynamics of the average parts velocity is decoupled from the evolution of the density. In particular, there exits Riemann data such that solutions to equation (6) lead to $\delta$-concentrations. The model 
is also not strictly hyperbolic since it has a coinciding eigenvalue $\lambda_{1}=\lambda_{2}=u$ and any solution to the Riemann problem is either a constant, a traveling contact discontinuities separated by vacuum or a singular measure in $\rho$. Neglecting the concentrations lead to a diagram consisting of only straight lines with slope $u_{\ell}$ and $u_{r}$, respectively, compare Figure 4. The presented model on the other hand also allows for smooth solutions in the case $Y_{\ell}=Y_{r}$ and $\rho_{\ell}>\rho_{r}$ and does not give rise to any concentrations in the density.

3.1. Particle interpretation of second-order model. The derived second-order model may be reinterpreted as a particle model by transformation to Lagrange (mass) coordinates. Those equations form a deterministic formulation of the underlying DES in terms of the particle density and the average velocity. Besides the modeling point of view this also leads to equation (9) that may be used to derive suitable boundary conditions for equation (5).

We assume for the following computation $x \in \mathbb{R}$. Even so $u(x, t)$ is not a conserved variable we write the model for $(\rho, u)(X, t)$ in Lagrange coordinates $(X, t)$ to provide an explanation. Clearly, $z$ and $Y$ may be computed out of $(\rho, u)$ as $z=u \rho(1+\rho)$ and $Y=u(1+\rho)$, respectively. Note that contrary to the scalar model (1) and (2), the variables $\rho$ and $u$ are independent. In $(\rho, u)$ the system reads in Eulerian coordinates $(x, t)$ :

$$
\begin{aligned}
& \partial_{t} \rho+\partial_{x}(\rho u)=0, \\
& \partial_{t}(u(1+\rho))+u \partial_{x}(u(1+\rho))=0 .
\end{aligned}
$$

The Lagrange coordinates $[12,35]$ are defined by

$$
X=X(x, t)=\int^{x} \rho(\xi, t) d \xi, \quad t=t
$$

and

$$
\partial_{t} X=-(\rho u), \quad \partial_{x} X=\rho .
$$

Hence, conservation of mass is given by $\partial_{t} \partial_{x} X=\partial_{x} \partial_{t} X$. Next, we introduce the density $\bar{\rho}$ and the velocity $\bar{u}$ in Lagrange coordinates as

$$
\rho(t, x)=\bar{\rho}(t, X(x, t)), \quad u(t, x)=\bar{u}(t, X(x, t)) .
$$

A simple calculation yields that $(1+\rho) \partial_{t} u+u \partial_{x} u=0$ and the resulting equations for $(\bar{\rho}, \bar{u})$

$$
\partial_{t}\left(\frac{1}{\bar{\rho}}\right)-\partial_{X} \bar{u}=0, \quad \bar{u}(1+\bar{\rho})=C,
$$

where $C$ may depend on $X$ but not on $t$. Clearly, the second equation shows that $\bar{Y}=$ $\bar{u}(1+\bar{\rho})$ is a property of the product since it remains constant in time when transported with the flow, i.e., in Lagrange coordinates $(X, t)$. The quantity $\frac{1}{\bar{\rho}}$ has the unit of an average spacing. Hence, if we semi-discretize the previous equations in $X$ and denote the grid points by $X_{i}$ we obtain a labeling of the products by $X_{i}, i=1, \ldots, P$. Then, $X_{i+1}-X_{i}=\Delta X$. We write $\bar{\rho}\left(X_{i}, t\right)=\bar{\rho}_{i}(t)$ and similarly for $\bar{u}$. Due to the definition of $X$ we may view each grid point $X_{i}$ in Lagrangian coordinates as a generic product. For 
non-negative $\bar{u}$ we obtain

$$
\frac{d}{d t}\left(\frac{1}{\bar{\rho}_{i}(t)}\right)=\frac{\bar{u}_{i+1}(t)-\bar{u}_{i}(t)}{\Delta X}, \quad \bar{u}_{i}(t)=\frac{Y_{i}(0)}{1+\bar{\rho}_{i}(t)}
$$

for $C=Y_{i}(0)$. Let us denote by $x_{i}$ the position of product $i$ in Euclidean geometry. The distance between two particles $i$ and $i+1$ is $x_{i+1}-x_{i}$ and the local density around particle $i$ is computed by using the specific volume $\Delta X$

$$
\rho\left(x_{i}, t\right)=\frac{\Delta X}{x_{i+1}-x_{i}} .
$$

Denoting by $\rho_{i}(t)=\rho\left(x_{i}, t\right)$ and $u\left(x_{i}, t\right)=u_{i}(t)$, and using the definition of $\bar{\rho}$ and $\bar{u}$ we obtain from equation (8)

or

$$
\frac{d}{d t}\left(x_{i+1}-x_{i}\right)=u_{i+1}(t)-u_{i}(t), \quad u_{i}(t)=\frac{Y_{i}(0)}{1+\frac{\Delta X}{x_{i+1}-x_{i}}}
$$

$$
\frac{d}{d t} x_{i}(t)=u_{i}(t), \quad u_{i}(t)=\frac{Y_{i}(0)}{1+\frac{\Delta X}{x_{i+1}(t)-x_{i}(t)}}, i=1, \ldots, P .
$$

Note that we may have also written the equations in the form $\frac{d}{d t} x_{i}=u_{i}, \frac{d}{d t} Y_{i}=0$. However, the system (9) allows for the following interpretation in terms of a production line. Each product $i$ follows the classical dynamics of being moved forward in stage $x$ by the velocity $u_{i}$. As in the scalar model (1) and (2) the velocity of the product $u_{i}$ does not change during production which coincides with the fact that products may not influence the machine performance. Also, in a DES as soon as the product enters the production process the products exit time is computable given the state of the full system. Therefore, the product velocity remains constant. Note that the behavior in traffic or gas dynamics is different in this respect. Contrary, to the simpler scalar model (1) and (2) we observe that $u_{i}$ even so it remains constant throughout the production process it may change with respect to $i$. Those changes are given precisely by the value of $Y_{i}(0)$. Hence, the second-order model allows to assign a possibly different velocity to each product $i$ upon entering the production process. This yields the second boundary condition for system (3). Therefore, the information of the new variable $Y$ enters in fact only through its value at the boundary $x=0$. Clearly, if $Y_{i}(0)=Y^{*}$ independent of $i$, the model (3) reduces to the scalar model with capacity $\mu=Y^{*}$. Therefore, non-constant values of $Y_{i}$ are understood as modeling of a production line having product dependent capacities. We may also state this differently: upon entering the production line each product $i$ moves through the factory as in a classical M/M/1-queuing system having a capacity of $Y_{i}$. Hence, $Y_{i}-Y^{*}$ is a measure for the product dependent deviation of a standard production process having capacity $Y^{*}$ for the production process encountered by product $i$.

3.2. Discussion of boundary conditions in Eulerian coordinates. The system (5) allows for waves of non-negative speed only. Therefore at $x=0$ we need to prescribe two boundary conditions. Clearly,

$$
(\rho u)(x=0, t)=\lambda(t)
$$


is the natural boundary condition for the conservation of mass equation. We propose the following condition on $(z u)$, respectively on $Y$. We have that

$$
(z u)=(\rho u)\left(\frac{\rho u}{\rho}+(\rho u)\right) .
$$

Note that $(\rho u)(0, t)$ is given by the influx $\lambda(t)$, but $\rho(0, t)$ is unknown. In absence of any other information on the boundary conditions we proceed by approximating $\rho(0, t)$ as follows: In view of the discussion of the Lagrange form of the model we know that $Y=\frac{(z u)}{(\rho u)}$ describes changes in the capacity of the production. Typically, those changes are due to the load of the factory. The load is given by the total wip $W(t):=\int_{0}^{1} \rho(x, t) d x$. We therefore prescribe as second boundary condition $\Lambda(t)$ if $W(t) \neq 0$ :

$$
(z u)(0, t)=\Lambda(t):=\lambda(t)\left(\frac{\lambda(t)}{W(t)}+\lambda(t)\right) .
$$

In case of $W(t)=0$ we simply use $\Lambda(t)=\lambda^{2}(t)$. Hence, the left boundary conditions are determined by the current load of the factory introducing a modification of the current capacity according to

$$
Y(0, t)=\lambda(t)\left(1+\frac{1}{W(t)}\right)
$$

for $W(t) \neq 0$. This condition is still not well-defined due to the implicit dependence on $\rho(t)$. We therefore replace $W(t)$ by $W(t-)$ with $W(0-)=\int_{0}^{1} \rho_{0}(\xi) d \xi$ being the initial wip.

3.3. Relaxation model. The model (5) can be extended to a case where the production facility at some given time $\varepsilon$ adjusts the current capacity to a desired value of $\mu$. This may be incorporated in the previous model by introducing a relaxation term similar to [23] leading to the slightly extended equations (11) in conservative form:

$$
\begin{aligned}
& \partial_{t} \rho+\partial_{x}(\rho u)=0, \\
& \partial_{t} z+\partial_{x}(z u)=\frac{1}{\varepsilon}(\mu \rho-z), \quad \text { with } \quad z=u \rho(1+\rho) .
\end{aligned}
$$

Note that in the quasi-linear form it is easy to see that the term is a relaxation on $Y$, i.e.,

$$
\partial_{t} Y+u \partial_{x} Y=\frac{1}{\varepsilon}(\mu-Y) .
$$

Obviously, for small values of $\varepsilon$ and $\mu$ independent of $(x, t)$ we obtain $Y=\mu+O(\varepsilon)$ and $u=\mu(1+\rho)+O(\varepsilon)$ and therefore an approximation to the dynamics of the scalar model (1) and (2).

3.4. Relation to continuous models with buffers. From a modeling point of view it may be desirable to rewrite the derived model in semi-discretized form. For scalar models this interpretation has been given in [21]. Due to the non-negative eigenvalues of the flux we apply an Upwind semi-discretization by the method of lines of equation 
(5) on an equidistant grid with cell centers $x_{i}, i=1, \ldots, N$ and cell size $\Delta x$, such that $N \Delta x=1$ and $\rho_{i}(t)=\frac{1}{\Delta x} \int_{x_{i-\frac{1}{2}}}^{x_{i+\frac{1}{2}}} \rho(\xi, t) d \xi$.

$$
\partial_{t}\left(\begin{array}{c}
\rho_{i} \\
z_{i}
\end{array}\right)+\frac{1}{\Delta x}\left(f_{i}-f_{i-1}\right)=0, \quad f_{i}=\left(\begin{array}{c}
\rho_{i} u_{i} \\
z_{i} u_{i}
\end{array}\right), i=1, \ldots, N
$$

The flux $f_{0}$ is according to the previous discussion given by

$$
f_{0}=\left(\lambda(t), \lambda^{2}(t)\left(1+\frac{1}{W(t)}\right)\right)^{T} .
$$

Introducing a new variable for the first cell as $(q, Z)=\Delta x\left(\rho_{1}, z_{1}\right)$ and using the boundary condition leads to the coupled system

$$
\frac{d}{d t} q=\lambda-\rho_{1} u_{1}, \quad \frac{d}{d t} \rho_{i}=\frac{1}{\Delta x}\left(\rho_{i} u_{i}-\rho_{i-1} u_{i-1}\right), i=1, \ldots, N,
$$

and similar equations for $\left(Z(t), z_{i}(t)\right)$ omitted for brevity. Equation (12) may be interpreted as a rate equation for the inventory $q$ due to its unit of parts. Note that the formulation (12) is in particular suitable for future extensions to a network of production lines. Therein, the inflow $\lambda$ would be replaced by the total inflow from all connected prior production lines.

\section{NUMERICAL RESUltS}

The numerical solution of the scalar model (1), (2) and the second order model (5) are discretized using a first-order explicit finite volume method. The discrete flux is obtained using an Upwind discretization due to the non-negative characteristic speed. As before an equidistant discretization in space with grid points $x_{i}$ for $i=1, \ldots, N$ and distance $\Delta x=x_{i+1}-x_{i}$ is used. The cell average at the discrete time $t^{n}$ is denoted by $\rho_{i}^{n}=\frac{1}{\Delta x} \int_{x_{i}-\frac{\Delta x}{2}}^{x_{i}+\frac{\Delta x}{2}} \rho\left(\xi, t^{n}\right) d \xi$ and similarly for $u$ and $Y$. The time discretization is given by $t^{n}=t^{n-1}+(\Delta t)^{n-1}$ for all $n$ and where at each time step $(\Delta t)^{n}$ is obtained using the CFL condition $(\Delta t)^{n}=\min _{i=1, \ldots, u_{i}^{n} \neq 0}\left\{\frac{1}{2}, \frac{\Delta x}{u_{i}^{n}}\right\}$. The factor $\frac{1}{2}$ has been added to avoid the case of zero velocities. If not stated otherwise the initial data is $\rho(x, t=0)=\frac{1}{2}$ and $u(x, t=0)=\mu$.

The scheme is modified in order to treat the source term (11) by using a first-order splitting in time. First, we solve the conservation law (5) $\left(t^{n}, t^{n+1}\right)$ and then explicitly solve the system of ordinary differential equations $\partial_{t} \rho=0, \partial_{t} z=\frac{1}{\varepsilon}(\mu \rho-z)$. This approach avoids a modification of previous CFL condition.

All implementations are done in Matlab. For the DES we translated a standard DES code as discussed in the Appendix A into Matlab. Additionally, the production rate is fixed at $\mu=5$ and the time horizon is set to $T=10^{3}$. In all results the boundary condition at $x=0$ is chosen to be $(\rho u)(0, t)=\lambda(t)$ and $(z u)(0, t)=\Lambda(t)$.

Besides knowledge on the structure a motivation to use PDE based models to simulate transient queuing system is the reduced computational complexity compared to DES simulations. In order to quantify we record Matlab CPU times for our implementations performed on a standard Desktop PC. We consider a ramp-up and ramp-down scenarios 
discussed below in more detail and compare the computational times for the secondorder model for various spatial grids with DES simulations. Since the DES describes a stochastic process a single DES simulation is not sufficient to obtain information on aggregated quantities of the modeled system as obtained through the PDE. Therefore, in Table 1 we consider different number of simulations to be compared with different spatial resolutions of the PDE. Clearly, the DES computation time increases linearly with the number of runs and the CPU times for the PDE simulation increases as the grid is refined. We observe that a fine resolution of the second-order PDE is as expensive as roughly 25 DES simulations. The relative error of the PDE is below $1 \%$ for any grid resolution higher than 20 spatial points. Since the CFL condition depends on the velocity $u$ different temporal grids are required for ramp up and ramp down simulations.

\begin{tabular}{|l||l|l|l|l|l|}
\hline $\mathrm{N}$ & 5 & 10 & 20 & 40 & 80 \\
\hline ramp down & 1.7272 & 3.3946 & 6.3684 & 12.3503 & 24.4537 \\
ramp up & 2.0352 & 3.7179 & 7.2867 & 14.4310 & 29.3513 \\
\hline \hline runs & 5 & 10 & 25 & 50 & 100 \\
\hline ramp down & 4.9651 & 10.161 & 24.1237 & 48.3448 & 102.9073 \\
ramp up & 4.9229 & 9.6539 & 23.9623 & 48.8150 & 102.6155 \\
\hline
\end{tabular}

TABLE 1. Comparison of Matlab CPU times (in seconds) for the simulation of the second-order relaxation model with $N$ spatial grid points on $[0,1]$ and $\varepsilon=100$ and different inflow scenarios. The bottom part refers to the DES simulation where runs denote the number of DES simulations.

Besides possibly computational advantages we perform qualitative comparison between the scalar model and the derived system. At first, we consider a ramp-up scenario at which the inflow $\lambda(t)$ changes linearly increasing between two constants, the other one is a ramp-down scenario where the change happens in the opposite direction.

In the numerical implementation of the boundary condition section 3.2 we use at time $n$ the following condition discretizing the previous equation

$$
Y^{n+1}(x=0)=\lambda^{n+1}\left(1+\frac{1}{\max \left\{W^{n}, \delta\right\}}\right),
$$

where $\delta$ is set to $\frac{1}{2}$.

For the ramp-up and ramp-down scenario a comparison of the outflux $(\rho u)(1, t)$ for the scalar model as well as the system using different relaxation rates $\varepsilon$ is presented in Figure 5. As expected small relaxation values lead to a similar dynamic of the secondorder to the scalar model. A similar result is observed in the ramp-down case and shown in Figure 6. Note that the curves of the influx $\lambda(t)$ and the second-order model for $\varepsilon=10000$ coincide.

Finally, we present a comparison to a time-dependent DES for a ramp-up and a rampdown scenario. We implemented a simple $\mathrm{M} / \mathrm{M} / 1$ discrete event simulator as described in the Appendix A. For many parts, constant inflow and sufficiently large simulation time the DES yields the well-known flux $F$ and wip $\rho$ relation given by equation (2). We modify the DES by allowing the influx to change over time as in the previous simulations. We then record the average cumulative outflux $\frac{1}{t} \int_{0}^{t} F(s) d s$ obtained from the DES for 

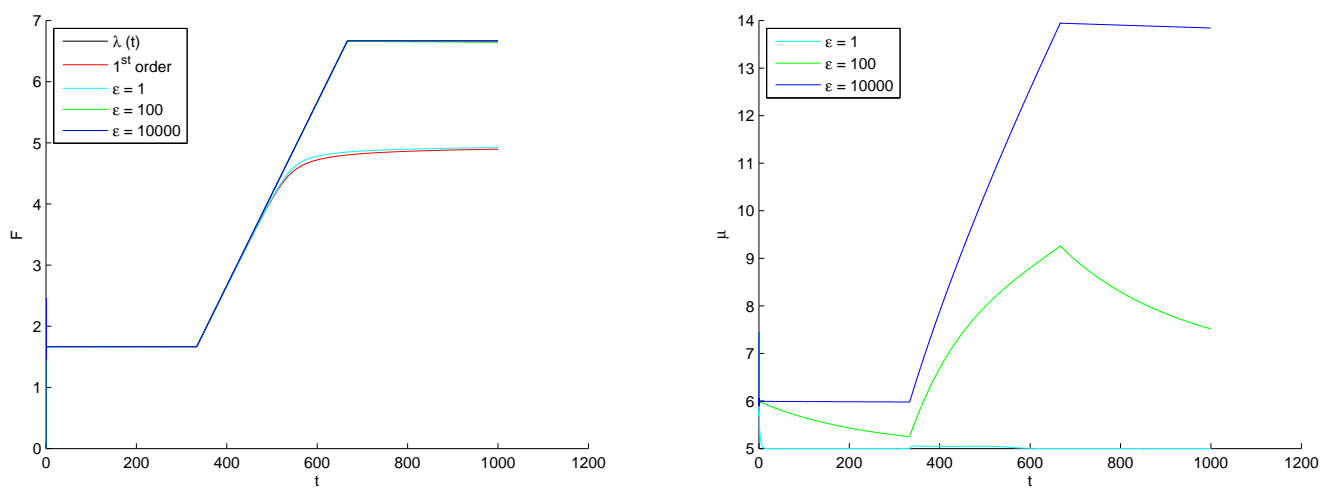

Figure 5. Left: Numerical simulation of the outflux $(\rho u)(1, t)$ for the scalar model (1) and (2) and the second order model (5) using the indicated relaxation rates $\varepsilon$. Right: Solution $Y(1, t)$ for different relaxation rates $\varepsilon$. The boundary condition at $x=0$ is given by $\lambda(t)$ and equation (10) for the second order model.
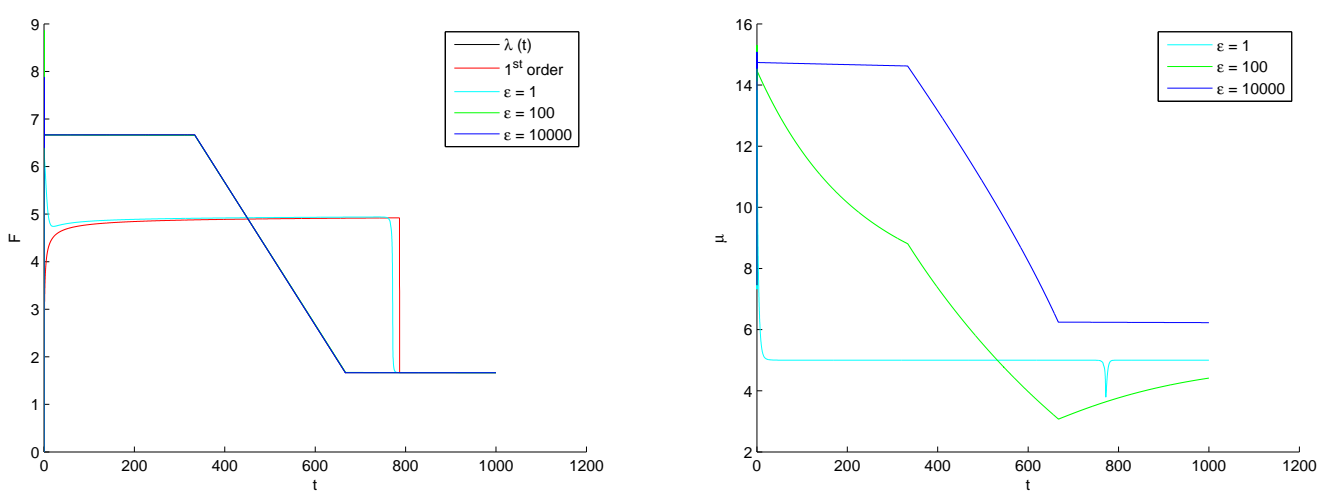

Figure 6. Left: Numerical simulation of the outflux $(\rho u)(1, t)$ for the scalar model (1) and (2) and the second order model (5) using the indicated relaxation rates $\varepsilon$. Right: Solution $Y(1, t)$ for different relaxation rates $\varepsilon$. The boundary condition at $x=0$ is given by $\lambda(t)$ and equation (10) for the second order model.

$t=0, \ldots, T$. The averaged cumulative outflux is then compared to simulations of the scalar model given by equation (1) and (2) and the second-order model (5), respectively. The results for a ramp-up scenario are depicted in Figure 7 and for a ramp-down scenario in Figure 8, respectively.

For every DES we obtain a data point indicated in both figures as green dots. The red line is the cumulative outflux using the scalar model. In particular, for the ramp-down scenario we observe that the scalar model underestimates the outflux generated by the DES. The simulation results for the second-order model are obtained using different 


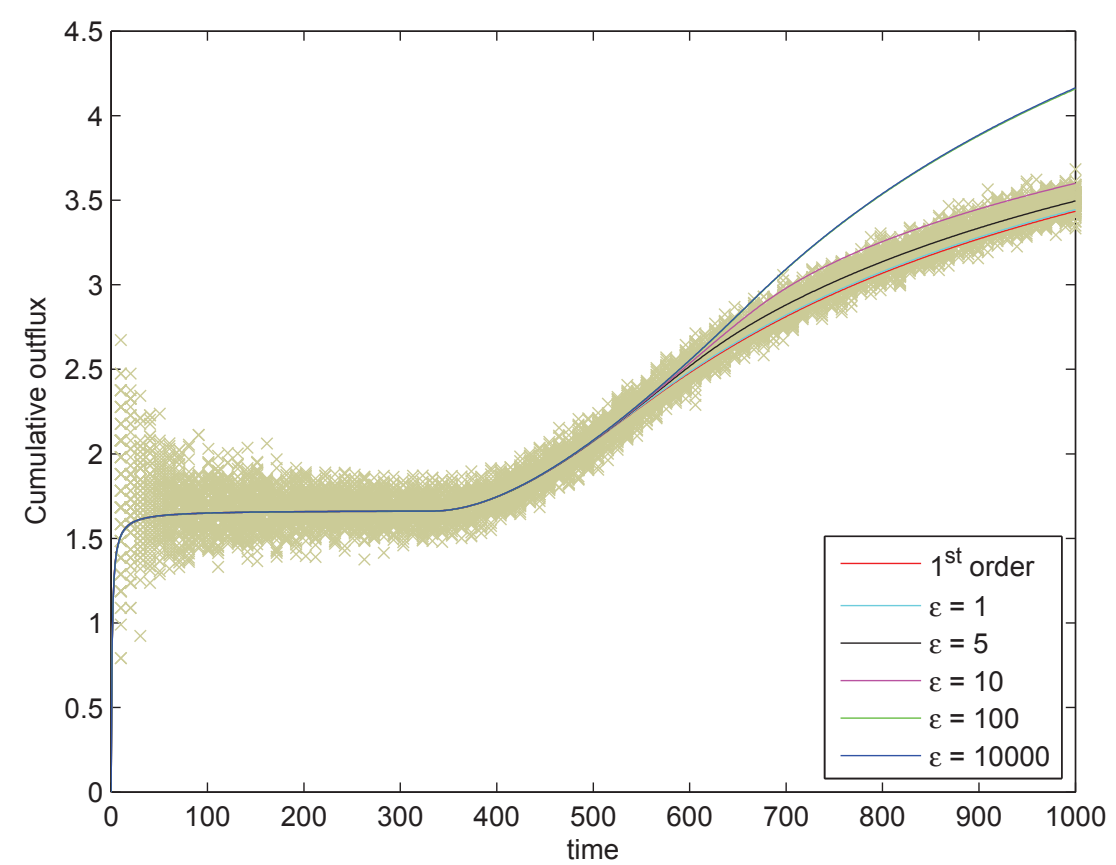

FiguRE 7. Numerical simulation of the cumulative outflux $\frac{1}{t} \int_{0}^{t}(\rho u)(1, s) d s$ for the scalar model (1) and (2) and the second order model (5) using the indicated relaxation rates $\varepsilon$. Data points are obtained for various simulations using the DES given in Appendix A for time dependent influx given by a ramp-up scenario.

relaxation rates $\varepsilon$. Clearly, for small values of the relaxation rate the prediction is close to the scalar model, however, for values of the relaxation of order ten, we obtain a better fit to the DES results compared with the scalar model. This provides numerical evidence that a single PDE might not be sufficient to capture the true DES dynamics. The effect is also visible in the ramp-up scenario but not as pronounced. Here, the scalar model already predicts the behavior of the DES. However, the second-order model for $\varepsilon=10$ yields a slightly better result for large times $t$.

Note that in the derivation of the scalar and second-order model only the machine capacity $\mu$ enters in the scalar model and in the relaxation term of the second-order model. It is therefore not immediate that the PDE (2) and (5) yields similar results. Figure 7 and Figure 8 show that the qualitative and quantitative behavior of time dependent DES are well-captured by the proposed models.

Finally, we present a combined ramp-up and ramp-down example in Figure 9. The observation for the combined example are similar to the simple ramp-up and ramp-down scenarios with a slightly too low predicted flow value for the scalar model. 


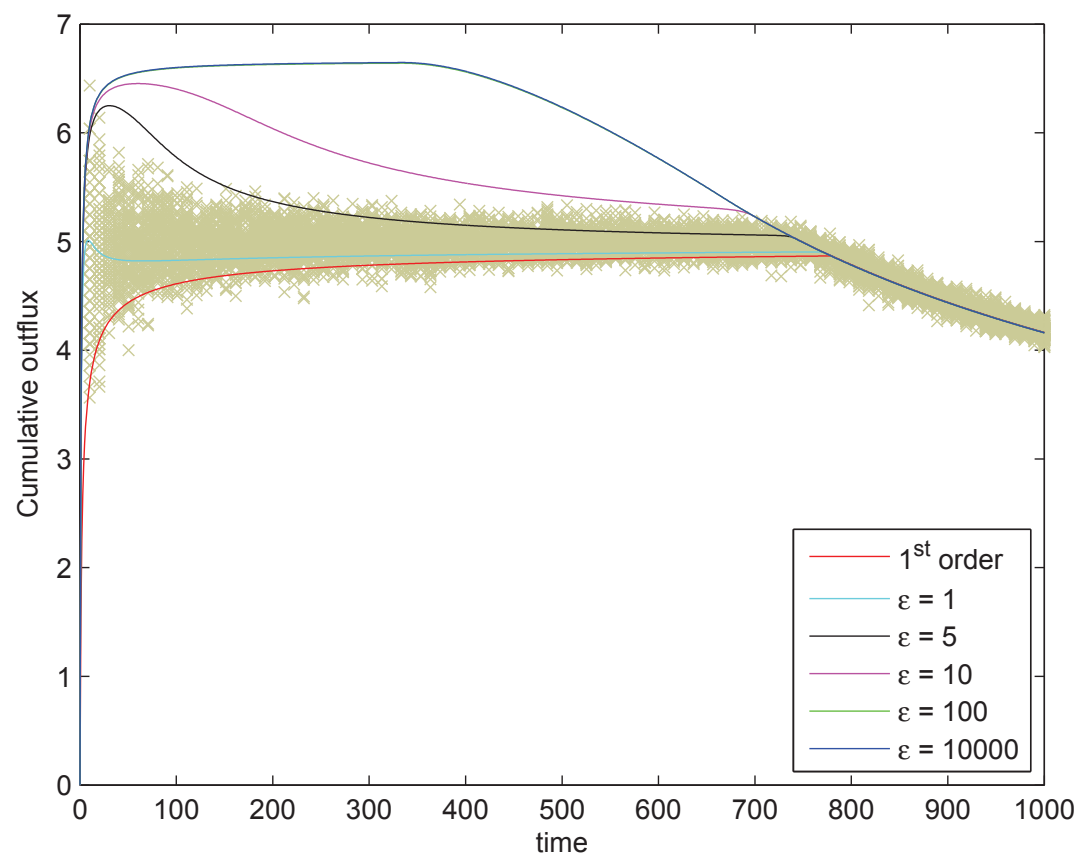

Figure 8. Numerical simulation of the cumulative outflux $\frac{1}{t} \int_{0}^{t}(\rho u)(1, s) d s$ for the scalar model (1) and (2) and the second order model (5) using the indicated relaxation rates $\varepsilon$. Data points are obtained for various simulations usind the DES given in Appendix A for time dependent influx given by a ramp-down scenario.

\section{Conclusion And Future Work}

In this work, we have developed first and second order macroscopic production models based on a discrete event simulator feeded with sampled data. The resulting models can be interpreted as an extension of classical $\mathrm{M} / \mathrm{M} / 1$ queuing theory models to transient regimes. However, the proposed procedure is not limited to $\mathrm{M} / \mathrm{M} / 1$ assumptions and can be extended to other distributions for the arrival and serving process. This would of course lead to other clearing functions.

As shown in our numerical experiments, the benefit of the second order model is that more information on the production process is available. Mathematically, the product information $Y$ is transported with the velocity of the products and is able to reproduce typical scalar conservation laws for production and more involved second-order trafficlike models, e.g., the Aw-Rascle-Zhang model, as well.

Various future work options are possible. For production purposes, usually complex systems are of interest meaning that different production steps are interlinked in the sense of a network. Suitable coupling conditions by installing buffers can be discussed for the second-order model. 

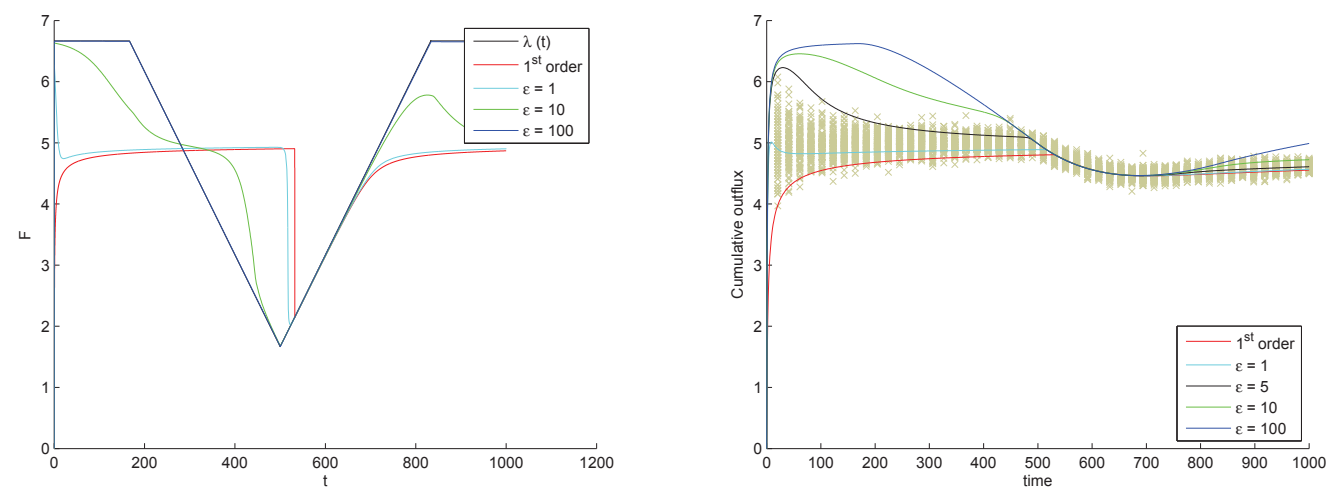

FiguRE 9. Right: Numerical simulation of the cumulative outflux $\frac{1}{t} \int_{0}^{t}(\rho u)(1, s) d s$ for the scalar model (1) and (2) and the second order model (5) using the indicated relaxation parameter $\varepsilon$. Data points are obtained for various simulations using the DES given in Appendix A. The influx is depicted as black line in the left diagram. Further, we show the total outflux for the scalar model and the system for relaxation times parameter $\varepsilon$ as indicated.

In the sense of optimal control questions concerning the overall performance may arise. The impact of second order production models is completely open at this point.

Acknowledgments. This work has been supported by the Cluster of Excellence 'Integrative Production Technology for High-Wage Countries' and the BMBF project KinOpt.

\section{Appendix A. A simple discrete event simulator}

The idea to write a discrete event simulator is taken from Figure 1.6 in Simulating Computer Systems, Techniques and Tools by M. H. MacDougall [29] except for sampling. The book introduces a discrete-event simulation language called smpl for $\mathrm{M} / \mathrm{M} / 1$-models and presents a $\mathrm{C}$ language implementation available for free download at http://ece.ut.ac.ir/Classpages/S86/ECE462/\#Software. The buffer policy is 'first-come, first-served'. We use the presented algorithms as example of a straightforward implementation. In the notation of the present paper the probability distribution for the interval times has mean $\lambda$ and the probability distribution for the production time has mean $\mu$. Then, for the inter-arrival times the density of the exponential probability distribution is given by $s \rightarrow \lambda \exp (-\lambda s)$. Running different realizations of the DES for fixed rates $\lambda$ and $\mu=5$ yields the result presented in Figure 1. This is the classical result in queuing theory and the expected relation of equation (2) between rate $\lambda=F$ and work-in-progress $W$ (or in this case equivalently $\rho$ ) is observed.

However, in Figure 7 and 8 the influx is chosen to be time-dependent $\lambda=\lambda(t)$ but the production rate $\mu$ is left constant. The detailled dependence of $\lambda$ on time $t$ is given in Section 4 . The smpl code can be easily modified to include time dependent rates $\lambda(t)$. 


\section{REFERENCES}

[1] Mobile millennium project: http://traffic.berkeley.edu.

[2] J. Abate And W. WhitT, Transient behavior of regulated brownian motion, $i$ : starting at the origin, Advances in Applied Probability, (1987), pp. 560-598.

[3] S. Amin, S. Andrews, S. Apte, J. Arnold, J. Ban, M. Benko, R. M. Bayen, B. Chiou, C. Claudel, C. Claudel, et Al., Mobile century using gps mobile phones as traffic sensors: A field experiment, (2008).

[4] D. Armbruster, P. Degond, and C. Ringhofer, A model for the dynamics of large queuing networks and supply chains, SIAM J. Applied Mathematics, 66 (2006), pp. 896-920.

[5] D. Armbruster, J. Fonteijn, And M. Wienke, Modeling production planning and transient clearing functions, Logistics Research, 5 (2012), pp. 133-139.

[6] D. Armbruster, S. Göttlich, And M. Herty, A scalar conservation law with discontinuous flux for supply chains with finite buffers, SIAM J. Appl. Math., 71 (2011), pp. 1070-1087.

[7] D. Armbruster, D. Marthaler, And C. Ringhofer, Kinetic and fluid model hierarchies for supply chains, Operations Research, 2 (2003), pp. 43-61.

[8] D. Armbruster, D. Marthaler, C. Ringhofer, K. Kempf, and T.-C. Jo, A continuum model for a re-entrant factory, Operations Research, 54 (2006), pp. 933-950.

[9] D. Armbruster and R. Uzsoy, Continuous dynamic models, clearing functions, and discreteevent simulation in aggregate production planning, in New Directions in Informatics, Optimization, Logistics, and Production, J. C. Smith, ed., vol. TutORials in Operations Research, INFORMS, 2012.

[10] J. Asmundsson, R. L. Rardin, C. H. Turkseven, And R. Uzsoy, Production planning with resources subject to congestion, Naval Res. Logist., 56 (2009), pp. 142-157.

[11] J. Asmundsson, R. L. Rardin, And R. Uzsoy, Tractable nonlinear production planning: Models for semiconductor wafer fabrication facilities, IEEE Transactions on Semiconductor Wafer Fabrication Facilities, 19 (2006), pp. 95-111.

[12] A. Aw, A. Klar, T. Materne, and M. Rascle, Derivation of continuum traffic flow models from microscopic follow-the-leader models, SIAM J. Appl. Math., 63 (2002), pp. 259-278.

[13] A. Aw And M. RAscle, Resurection of second order models of traffic flow, SIAM J. Appl. Math., 60 (2000), pp. 916-944

[14] J. Banks, J. S. Carson, And B. Nelson, Discrete-event system simulation, Prentice Hall International Series in Industrial and Systems Engineering, New Jersey, 1984.

[15] A. Bressan, S. Canic, M. Garavello, M. Herty, and B. Piccoli, Flow on networks: recent results and perspectives, European Mathematical Society-Surveys in Mathematical Sciences, 1 (2014), pp. $47-11$.

[16] C. D'Apice, S. Göttlich, M. Herty, and B. Piccoli, Modeling, simulation, and optimization of supply chains, Society for Industrial and Applied Mathematics (SIAM), Philadelphia, PA, 2010. A continuous approach

[17] C. D'Apice And R. Manzo, A fluid dynamic model for supply chains, Network and Heterogenous Media, 3 (2006), pp. 379-398.

[18] C. D'Apice, R. MAnzo, And B. PiCCOli, Modelling supply networks with partial differential equations, Quart. Appl. Math., 67 (2009), pp. 419-440.

[19] S. FAn, M. Herty, AND B. SEIBOLD, Comparative model accuracy of a data-fitted generalized Aw-Rascle-Zhang model, Netw. Heterog. Media, 9 (2014), pp. 239-268.

[20] S. Göttlich, M. Herty, And A. Klar, Network models for supply chains, Commun. Math. Sci., 3 (2005), pp. 545-559.

[21] S. Göttlich, M. Herty, And C. Ringhofer, Optimization of order policies in supply networks, European J. Oper. Res., 202 (2010), pp. 456-465.

[22] S. C. Graves, A tactical planning model for a job shop, Operations Research, 34 (1986), pp. 522 533.

[23] J. GreenberG, Congestion redux, SIAM J. Appl. Math., 64 (2004), pp. 1175-1185.

[24] J. M. Greenberg, Extensions and amplifications of a traffic model of Aw and Rascle, SIAM J. Appl. Math., 62 (2001/02), pp. 729-745 (electronic). 
[25] F. Guillemin And J. Boyer, Analysis of $M / M / 1$ queue with processor sharing via spectral theory, Queueing Syst., 39 (2001), pp. 377-397 (2002).

[26] M. Herty, A. Klar, And B. Piccoli, Existence of solutions for supply chain models based on partial differential equations., SIAM J. Math. Anal., 39 (2007), pp. 160-173.

[27] U. S. KARMARKAR, Capacity loading and release planning with work-in-progress (wip) and leadtimes, Journal of Manufacturing and Operations Management, 2 (1989).

[28] J.-P. LeBAcQue, Les modeles macroscopiques du traffic, Annales des Ponts., 67 (1993), pp. $24-45$.

[29] M. H. MacDougall, Simulating Computer Systems, Techniques and Tools, MIT Press series in Computer Systems, Cambridge, 1987.

[30] H. Missbauer, Order release and sequence-dependent setup times, International Journal of Production Economics, 49 (1997), pp. 131-143.

[31] H. Missbauer, Aggregate order release planning for time varying demand, Int. J. Production Research, 40 (2002), pp. 699-718.

[32] H. Missbauer, Models of the transient behaviour of production units to optimize the aggregate material flow, International Journal of Production Economics, 118 (2009), pp. 387-397.

[33] S. Moutari, M. Herty, A. Klein, M. Oeser, V. Schleper, and B. Steinauer, Modeling road traffic accidents using macroscopic second-order models, IMA Journal of Applied Mathematics, (2012).

[34] A. R. Odoni AND E. Roth, An empirical investigation of the transient behavior of stationary queueing systems, Operations Research, 31 (1983), pp. 432-455.

[35] D. H. WAGneR, Equivalence of the Euler and Lagrangian equations of gas dynamics for weak solutions, J. Differential Equations, 68 (1987), pp. 118-136.

[36] H. M. Zhang, A non-equilibrium traffic model devoid of gas-like behaviour, Transportation Research Part B, 36 (2002), pp. 275-298.

(L. Forestier-Coste) RWTH Aachen University

E-mail address: forestier@igpm.rwth-aachen.de

(S. Göttlich) University of Mannheim

E-mail address, Corresponding author: goettlich@uni-mannheim.de

(M. Herty) RWTH AACHEN UNIVERsity

E-mail address: herty@igpm.rwth-aachen.de 